\title{
Climate change dialogues
}

\author{
Human influence on the planet is undeniable. Making a switch from exploitation to maintenance of \\ natural resources depends on a step change in communication, to convince the Earth's population of the \\ necessity for a fundamental change of course.
}

The idea of the Anthropocene is a powerful concept. The term aptly conveys the notion that humankind has emerged as a force for change on a planetary scale - regardless of whether it will be formally confirmed as a geological era. There is no inherent reason why this force should be for the worse. But, so far, attempts to steer the world's population towards sustainable use of the Earth's natural resources have largely failed.

The conference 'Planet under Pressure' (http://www.planetunderpressure2012.net/), held in London from 26-29 March 2012, attempted another nudge in the direction of sustainability. Only time will tell how successful the meeting was in reaching this goal. But a common thread ran through the sessions and conversations that may open avenues for progress: the necessity of establishing a dialogue, in the full bidirectional sense of the word, between the scientific sphere and the rest of society.

That it is important for climate researchers to talk about their insights on climate warming, sea-level rise, ice melt and changes in the water cycle has been brought home in the past few years, in the face of vigorous public debate. Many scientists now realize that a lack of outward communication leaves a void in the public discourse that will only be filled by nonscientific voices. But development of a return flow of information - of scientists listening to the decision-makers as well as the public - is still in its infancy.

This return flow is essential if science is to inform policy. To frame scientific findings in a way that will be heard and understood, scientists have to know their audience their worries, questions, preconceptions and interests. The message may also need to be adapted in language and level, depending on who is being addressed. An understanding of where the concerns of the public and decision-makers lie will not only aid communication, but could also help to shape directions of research.

There are instances where public concerns have entered the scientific debate. For example, following the European heatwave in 2003, people wanted to know whether climate change was responsible for this extreme event. From a scientific

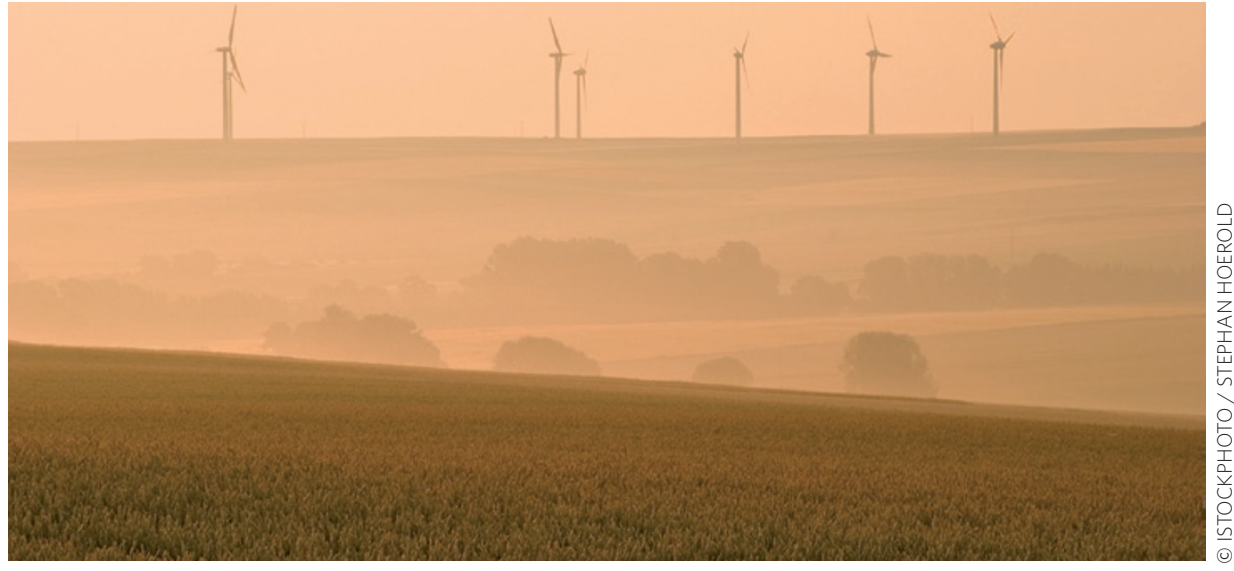

point of view, this question is not well posed - natural variability always contributes to individual extreme events, so full responsibility can never be allocated. Nevertheless, scientists took up the challenge to get as close to a useful answer as they could. They offered the conclusion that because of human influence, a European heatwave of the observed magnitude was at least twice as likely as it would have been in the absence of human-induced warming (Nature 432, 610-614; 2004).

The role of global warming in heatwaves and other climate extremes has attracted further attention, not least because of public interest. For example, two groups studied the Russian heatwave in summer 2010 that led to tens of thousands of deaths, as well as substantial economic losses - but arrived at apparently conflicting conclusions. One study suggested that the magnitude of the event was mainly natural in origin (Geophys. Res. Lett. 38, L06702; 2011). The other concluded that a record-breaking heatwave in Russia in 2010 was five times more likely as a result of climate warming (Proc. Natl Acad. Sci. 108, 17905-17909; 2011). Yet, in a later study, the two statements were found to be reconcilable (Geophys. Res. Lett. 39, L04702; 2012): although most of the anomalous warmth was a result of natural atmospheric dynamics, the risk of such a record-breaking event was probably multiplied because of the background warming.

Critically, although the studies are not necessarily contradictory, they convey very different messages. The case illustrates the difficulty faced by scientists when informing policy. It is not enough that any statements made are correct. Equally importantly, the bottom-line conclusion that will eventually settle in the public mind also needs to reflect scientific understanding. To achieve such a level of communication, it is essential to understand the societal context into which such messages will fall, and to be aware that this context can sometimes be far removed from the scientific discourse.

It would be good for scientists to actively seek out opportunities to listen to the concerns and questions of the public. Media training to improve communication skills is good, but it may be even better to participate in question-and-answer radio shows, engage with people in social networks, or simply reach out to local schools.

Climate researchers discuss their work mostly with their peers - and so they should, in the interest of scientific progress. However, as a result, their intake of viewpoints is skewed towards those who agree on, and are aware of, the basics of climate dynamics. This makes it easy to forget the limits of common knowledge and beliefs in society as a whole.

To redress the balance, climate scientists should make every effort to expose themselves to the questions of their intended audiences - be it politicians, journalists or the general public. Eventually, listening will help scientists to communicate more effectively, and may spark ideas for societally relevant research. 